Received: 2020/09/15, Revised: 2020/11/13, Accepted: 2020/12/04, Published: 2020/12/31 (๑2020 Hae-Sung Cho et al:; Licence Physical Activity and the terms of the creative commons attribution license (https:/l creativecommons.org/licenses/by-nc/2.0/), which permits unrestricted use, distribution, and reproduction in any medium, provided the orginal work is properly cited.

${ }^{*}$ Corresponding author : Hyo Youl Moon

Department of Physical Education, Seoul National University, Seoul, Republic of Korea.

Tel: +82-2-880-7802

E-mail: skyman19@snu.ac.kr

@2020 The Korean Society for Exercise Nutrition Nutrition. This is an open access article distributed under

\section{Lactate consumption mediates repeated high-intensity interval exercise-enhanced executive function in adult males}

\author{
Hae-Sung Cho ${ }^{1}$ / Won Sang Lee ${ }^{1}$ Kyeong Jin Yoon ${ }^{1}$ / \\ Soo Hong Park ${ }^{1}$ / Hyung Eun Shin ${ }^{2}$ / Yeon-Soo Kim ${ }^{1,3}$ / \\ Hyukki Chang ${ }^{5}$ / Hyo Youl Moon ${ }^{1,3,4^{*}}$ \\ 1. Department of Physical Education, Seoul National University, Seoul, Republic of Korea \\ 2. Health and Exercise Science Laboratory, Seoul National University, Seoul, Republic of Korea \\ 3. Institute of Sport Science, Seoul National University, Seoul, Republic of Korea \\ 4. School of Biological Sciences, Seoul National University, Seoul, Republic of Korea \\ 5. Department of Human Movement Science, Seoul Women's University, Seoul, Republic of Korea
}

\section{INTRODUCTION}

Lactate is a principal energy substrate for interval exercise (HIIE) can increase the blood lactate level, brain lactate uptake, and executive function (EF). However, repeated HIIE can attenuate exerciseinduced increases in lactate level and EF. The lactate levels in the brain and blood are reported to be correlated with exercise-enhanced EF. However, research is yet to explain the cause-and-effect relationship between lactate and EF. This study examined whether lactate consumption improves the attenuated exerciseenhanced EF caused by repeated HIIE.

[Methods] Eleven healthy men performed two sets of HIIE, and after each set, 30 min were given for rest and examination. In the $2^{\text {nd }}$ set, the subjects consumed experimental beverages containing $(n=6)$ and not containing $(n=5)$ lactate. Blood, cardiovascular, and psychological variables were measured, and EF was evaluated by the computerized color-word Stroop test.

[Results] The lactate group had a higher EF $(P<$ $0.05)$ and tended to have a higher blood lactate level $(P=0.082)$ than the control group in the $2^{\text {nd }}$ set of HIIE. Moreover, blood lactate concentration was correlated with the interference score (i.e., reverse score of EF) $(r=-0.394 ; P<0.05)$

[Conclusion] Our results suggest that the attenuated exercise-enhanced EF after repeated HIIE can be improved through lactate consumption. However, the role of lactate needs to be elucidated in future studies, as it can be used for improving athletes' performance and also in cognitive decline-related clinical studies.

[Key words] Exercise, Performance, Sports drink, Fatigue, Brain energy, Cognitive function

[Abbreviations] EF, executive function; HIIE, highintensity interval exercise; PAR-Q+, physical activity readiness questionnaire for everyone; BDNF, brainderived neurotrophic factor; CWST, color-word Stroop test
The second half of a sporting event is the time when players' performances weaken and many points are conceded. The time-scoring statistics of the FIFA World Cup (1930-2018) suggest that, on an average, more goals are scored in the second half than in the first half of the game. For example, in the quarter-final of the World Cup in 2018, the six teams scored $65 \%$ of their goals during the second half ${ }^{1}$. Moreover, the performance of the referees, who run with the players throughout the game, also declines in the second half. During the official matches of the Brazilian Under-20 Championship (U-20) in 2002, of a total of 321 foul calls recorded, missed calls were more frequent in the second half $f^{2,3}$.

Many studies have focused on endured physical function for improving the performance of players during the second half of matches. In addition to physical function, optimal cognitive function is also required to maintain the performance of players until the end of the game. Some studies have observed a positive correlation between performance and the executive function (EF) score, an indicator of cognitive function ${ }^{4,5}$. Vestberg et al. ${ }^{6}$ suggested that $\mathrm{EF}$ is a determining factor for players with higher performance. EF, which includes working memory, cognitive flexibility, and inhibitory control, is a part of the essential cognitive processes for behavioral control ${ }^{5}$. Many brain sites, such as the dorsolateral prefrontal, anterior cingulate, and parietal cortex, are related to EF. EF is usually evaluated using the Stroop test ${ }^{7,8}$, one of the most commonly used tests in neuropsychology and various fields of study ${ }^{8}$.

The decreased performance of players during the second half of matches is related to fatigue ${ }^{9}$. From a physiological perspective, fatigue is divided into two components: central fatigue and peripheral fatigue. Central fatigue is related to reduced cognitive performance, represented by the decline in cognitive function caused by changes in the concentration of neurotransmitters in the synapses of the central nervous system $(\mathrm{CNS})^{10,11}$. Moreover, central fatigue affects not only cognitive function but also muscle contraction, which induces a decline in physical function; therefore, overall performance can be affected ${ }^{10}$.

The energy depletion model is one of the main theories in fatigue re- 
search $^{12}$. Central fatigue is also related to energy depletion in the CNS, as neurons in the CNS require energy for the transport of neurotransmitters and ions for neural activi$\mathrm{ty}^{7}$. Thus, decreased cognitive function induced by central fatigue can be explained by the energy metabolism of the CNS, particularly within the brain. Blood-borne glucose is the main fuel for the human brain at rest. However, when the lactate concentration in the blood is increased during exercise, it replaces glucose as the primary energy source for the brain ${ }^{7,13}$. Lactate is an efficient fuel for brain energy metabolism and neurons preferably use lactate during exercise, even when both glucose and lactate are present ${ }^{14-16}$. Furthermore, as exercise intensity increases, brain glucose uptake decreases and brain lactate uptake increases in proportion to the blood lactate level ${ }^{16-18}$.

Previous studies have reported correlations between lactate and EF. A single bout of high-intensity interval exercise (HIIE, between $60 \%$ and $90 \%$ of peak $\mathrm{VO}_{2}$ ) increases the level of blood lactate and EF compared to moderate-intensity continuous exercise $\left(60 \% \text { of peak } \mathrm{VO}_{2}\right)^{19}$. Furthermore, a single HIIE bout can increase the level of blood lactate, brain lactate uptake, and EF. However, repeated HIIE can attenuate exercise-induced increases in lactate level and EF. ${ }^{7,20}$. Therefore, the correlation between lactate and EF seems to be associated with the relationship between blood lactate level ${ }^{21}$ and decreased athletic performance ${ }^{2,22}$ in the second half of the game. Therefore, it is necessary to further verify the relationship between lactate and EF.

However, the positive correlation between lactate and EF alone cannot explain the causality, as several studies have shown that exercise changes multiple psychological factors and the level of brain-derived neurotrophic factor (BDNF), which are also related to cognitive function ${ }^{7,23-27}$. Furthermore, relatively few studies have attempted to confirm the improvement of cognitive function through the consumption of lactic acid in humans, although Holloway et al. have ${ }^{28}$ reported cognitive recovery through lactate injections after traumatic brain injury in rats. Therefore, the purpose of this study was to identify whether lactate consumption affects the maintenance of exercise-induced improvement in EF.

\section{METHODS}

\section{Participants}

Eleven healthy adult males were assigned to one of two study groups (lactate group: $n=6$; control group: $n=5$ ). Smokers and individuals with neuromuscular, neurological, metabolic, cardiovascular, respiratory, and visual disorders were excluded. The participants who had body mass in$\operatorname{dex}>18.5 \mathrm{~kg} / \mathrm{m}^{2}$ and $<35 \mathrm{~kg} / \mathrm{m}^{2}$ and were able to exercise were selected through the 2019 Physical Activity Readiness Questionnaire for Everyone (PAR-Q+ ${ }^{29}$. Prior to the experiment, all the procedures of the experiment were explained to the participants and they signed a written informed consent.

\section{Procedure and conditions}

All the procedures were approved by the ethics commit- tee of Seoul National University (IRB No. 1909/002-010). For each experiment, the participants made the following two visits:

\section{Screening visit}

The participants received an explanation of the experimental protocol and procedures. To determine the exercise intensity of the participants accurately, they underwent graded exercise testing $(<$ Exercise intensity setting protocol $>$ ) as presented below. The maximal workload for each participant was calculated. After completion of the preexaminations, the participants were asked to refrain from introducing any drastic changes in their normal routine for a week. Additionally, prior to the test day, the researchers contacted the subjects to remind them to avoid consuming caffeine and alcohol and performing strenuous exercise. To progress to a double-blind randomized control model, the principal investigator asked a third person not related to the experiment to make different types of experimental beverage for the two groups and to randomly assign subjects to the lactate and control groups.

$<$ Exercise intensity setting protocol $>$

1. A 5-min-long preparation time was provided when the subject arrived in the measuring room.

2. The workload of the cycle ergometer was set at $30 \mathrm{~W}$; the subject kept pedaling for $3 \mathrm{~min}$ at $60 \mathrm{rpm}$.

3 . The load was increased by $15 \mathrm{~W}$ every minute. If 60 rpm was not maintained for more than $30 \mathrm{~s}$, the previous highest level of workload was considered as the subject's exercise intensity.

\section{Experimental visit}

Before starting the experiment, the subjects were reminded about the experimental protocol. To exclude learning effects, the subjects practiced the Stroop test five times to familiarize themselves with the tests. After body measurement, the following measurements were conducted:

1. Felt arousal scale, visual analog scale, peripheral oxygen saturation, and pulse rate; 2. Blood glucose and lactate levels; 3. Color-word Stroop test; and, 4. Blood BDNF level

Subjects performed the two sets of HIIE following the $<$ High Intensity Interval Exercise Program $>$ as presented below. After the first HIIE, a rest period of 40 min was given. Later, the tests were performed again immediately after HIIE, and after $10 \mathrm{~min}, 20 \mathrm{~min}$, and $30 \mathrm{~min}$ after the exercise. After the first exercise protocol and rest period, the second exercise protocol was initiated. Ten minutes after the start of the second HIIE protocol, the subjects consumed their experimental beverages within a minute, and the tests were performed in a similar manner as that in the first HIIE protocol.

\section{$<$ High-intensity interval exercise program $>$}

1. A 5-min-long preparation time was provided when the subject arrived in the measuring room. 
2. The cycle ergometer was set at $50 \%$ of the subject's maximum workload and the subject was asked to perform a 2 min warm-up at $60 \mathrm{rpm}$.

3. After the 2-min warm-up exercise, the exercise intensity was set to $90 \%$ of the subject's maximum work max and the subject exercised for $3 \mathrm{~min}$ at $60 \mathrm{rpm}$.

4. After $3 \mathrm{~min}$ of exercising, the intensity was set at 50\% of the subject's set maximum work max and the subject performed the exercise for $2 \mathrm{~min}$ at $60 \mathrm{rpm}$.

5 . The steps 3 and 4 were performed alternately three more times (last moderate-intensity exercise for $2 \mathrm{~min}$ was the cool-down exercise).

\section{Body measurement}

The subjects stood on the marked foot shape of a manual extensometer, stretching their spine, inhaling deeply, and exhaling one-third of the time, after which they were measured with suppressed breathing. Body composition measurements, such as weight, skeletal muscle mass, lean mass, body fat percentage, and waist-hip ratio were measured using Inbody 720 (Biospace, Korea). To ensure accurate measurement, the participants were asked to remove unnecessary accessories and clothing before the measurements were taken. In addition, the subjects were contacted one day before the measurement and asked to refrain from vigorous exercise, alcohol, and caffeine, and eating any food other than normal meals.

\section{Blood lactate}

Blood lactate levels were analyzed using Lactate Pro 2 \& strip (LT-1730, Arkray, Japan). When the subjects sat on chairs and relaxed, the experimenters wiped their fingertips with alcohol swabs for disinfection, pricked a finger using a lancing device (Accu-Chek Softclix Plus lancing device \& Softclix, Roche ${ }^{\circledR}$, Germany), and placed the blood onto the test strip. In this way, blood lactate was measured five times for each set (a total of 10 times), that is, pre-exercise, postexercise, and 10, 20, and 30 min post-exercise.

\section{Other measurement variables}

Cardiovascular and blood variables, BDNF, and psychological variables were all evaluated, as explained in the Supplementary Materials and Methods.

\section{Color-word Stroop test}

The color-word Stroop test (CWST) is one of the most commonly used neuropsychological tests to evaluate EF, especially selective attention and inhibitory control ${ }^{8}$. In this study, the computer-based CWST involved three types of tasks and four colors (red, green, blue, and yellow). The congruent task was the first type of CWST, in which the text was the same in meaning and color, and the color of the text was selected. The neutral task was a test that selected the color of the rectangle. The incongruent task was a test in which the text was different in meaning and color, and the color of the text was selected. The answers to the questions were provided as 1, 2, 3, and 4, and the subjects were asked to place their index and middle fingers on the numbers 1, 2, 3, and 4 of the keyboard. Twelve questions of each type were presented, three types of tests were randomly assigned, and continuing with three or more of the types and answers was prohibited. The total reaction time(RT), accuracy rate, and interference score were measured, and the formula used for calculating the interference score ${ }^{7}$ was as follows:

Interference score $=\frac{(\text { RT of incongruent task }-R T \text { of neutral task })}{R T \text { of neutral task }} * 100$

We encountered an error in which the response time was measured at an abnormally fast rate due to two mechanical problem-induced inputs during the examination. To correct for this, one question recorded in the minimum response time was removed from all the types, and the results of the 33 questions were used. The CWST was conducted in a separate place that was curtained off and with minimal noise disturbance. The CWST software was provided by the Department of Human Movement Science (Seoul Women's University).

\section{Experimental beverage}

To identify the effects of lactate consumption on the maintenance of decreased EF and the concentration of blood lactate after repeated HIIE, an experimental beverage was provided to each group $10 \mathrm{~min}$ after the $2^{\text {nd }}$ HIIE started. A lactate drink was provided to the lactate group that was composed of a "food grade" $88 \%$ lactic acid solution (LD Carlson Company, USA), which is mainly used beer manufacturing. Given the insufficiency of advanced empirical studies on the timing and concentration of lactate to be administered, a pilot test was conducted. The overall process for the test is shown in Supplementary Materials and Methods, and the results are presented in Supplementary Results. Experimental beverages were produced based on the results, as shown in Supplementary Tables S1 and S2. To set the total volume of the solution for the control group, a sports drink (Pocari Sweat ${ }^{\circledR}$, Otsuka Pharmaceutical, Japan) was provided at $3.5 \mathrm{ml}$ per weight $(\mathrm{kg})$ of the subject. The experimental beverage for the lactate group was made following these procedures. First, the total volume of the solution was set to equal that of the control group; then, $1 / 1000$ of the total calculated volume (about $0.1 \%$ concentration) was added to the experimental beverage and the remaining volume was filled with the sports drink, except for the amount of lactic acid solution added in the total amount. Overall information about the composition and volume of the experimental beverage is presented in Supplementary Table S3.

From the results of the pilot test (Supplementary Tables S1 and S2), ingestion timing was based on the expectation that the blood lactate levels would rise 10 to $20 \mathrm{~min}$ after the ingestion of lactate. Therefore, experimental drinks were given to the participants $10 \mathrm{~min}$ after the start of the $2^{\text {nd }}$ HIIE.

\section{Statistical analysis}

All data were analyzed using IBM SPSS statistics 25 and Prism 7 software. Nonparametric tests were used as the 
number of samples in this study was less than 10 per group, and the normality and variability assumptions were not met. Thus, instead of the three-way repeated measures ANOVA, which was deemed most suitable for this study, the variations in time, group, and set were analyzed, and the interaction effects of the three variables were excluded.

General characteristics such as age, height, and weight of the subjects were described using descriptive statistics. To test the homogeneity between the two groups, the difference between the general characteristics of each group and the time points of the remaining measurement variables were analyzed using the Mann-Whitney test. Friedman test was conducted to check the time-induced change within each group by set, after which Dunn's multiple comparison test was conducted as a post-hoc test to compare the preand post-exercise recovery periods in each set $(10,20$, and 30 min after HIIE). In order to identify the effect of lactate consumption, the differences in measurement variables between groups in the $2^{\text {nd }}$ HIIE set and the variations over the set within each group $\left(1^{\text {st }}\right.$ set $-2^{\text {nd }}$ set values $)$ were compared over time using the Mann-Whitney test. Spearman's correlation test was used to analyze the correlation between the results of the CWST and the variables. Statistical significance was set to $\alpha=0.05$, and the data are expressed as the means and standard errors of the means (Mean \pm SEM).

\section{RESULTS}

\section{General characteristics}

The general characteristics of the two groups and the results of the homogeneity test are presented in Table 1 . The two-tailed independent t-test showed no significant differences in the general characteristics between the two groups $(P>0.05)$.

\section{Blood lactate level}

The overall changes in the blood lactate levels of the groups are presented in Figure 1. The changes in blood lactate levels over time in each group are shown in Supplementary Table S4. The control group had a significantly different blood lactate level over time during the first $(P<$ $0.001)$ and second sets $(P<0.01)$. The post-hoc analysis in-

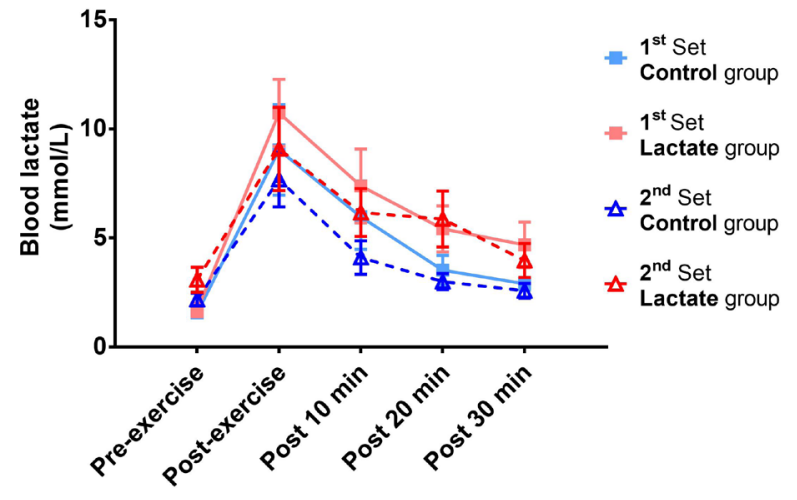

Figure 1. Changes in blood lactate levels; the differences in the inter-group blood lactate levels in the $2^{\text {nd }}$ HIIE set and the variation over the set within each group $\left(1^{\text {st }}\right.$ set $-2^{\text {nd }}$ set value $)$ were compared over time using a Mann-Whitney test. Friedman test was conducted to check the time-induced change within each group by set. Data are expressed as the means \pm standard errors of the means.

dicated significant increases in the post-exercise $(P<0.001)$ and 10 min post-exercise $(P<0.05)$ levels as compared to the pre-exercise levels in the first set. Additionally, there was a significant increase in the post-exercise lactate levels $(P<0.001)$ as compared to the pre-exercise lactate levels in the second set.

There were significant differences over time in both the first and second sets of lactate groups $(P<0.001)$. The posthoc analysis indicated a significant increase in the post-exercise $(P<0.001)$ and 10 min post-exercise $(P<0.05)$ levels as compared to the pre-exercise level in the first set. In the second set, unlike the control group, there was a significant increase post exercise $(P<0.001)$ and 10 min post exercise $(P<0.05)$.

Blood lactate levels were compared between the two groups by time point, and the amount of blood lactate level changes were compared by set (Table 2). There were no significant differences in the blood lactate levels between the two groups $(P>0.05)$. However, at $10 \min (P=0.0823)$ and 20 min after the $2^{\text {nd }} \operatorname{HIIE}(P=0.0974)$, the lactate group had higher blood lactate levels than the control group, although this difference was not significant. Additionally, on

Table 1. General characteristics of the two groups and homogeneity tests

\begin{tabular}{cccc} 
General characteristics & $\begin{array}{c}\text { Control group } \\
(\boldsymbol{n}=\mathbf{5})\end{array}$ & $\begin{array}{c}\text { Lactate group } \\
(\boldsymbol{n}=\mathbf{6})\end{array}$ & $\begin{array}{c}\boldsymbol{P} \text { value } \\
\text { Age }\end{array}$ \\
$26.4 \pm 1.03$ & $24.17 \pm 1.869$ & 0.253 \\
\hline Height $(\mathrm{cm})$ & $176.7 \pm 1.197$ & $176 \pm 2.715$ & 0.818 \\
\hline Body weight $(\mathrm{kg})$ & $81.06 \pm 2.586$ & $73.67 \pm 4.005$ & 0.126 \\
Skeletal muscle & $37.16 \pm 0.975$ & $34.25 \pm 2.193$ & 0.329 \\
mass $(\mathrm{kg})$ & $15.9 \pm 2.562$ & $13.33 \pm 1.534$ & 0.628 \\
\hline Body fat mass $(\mathrm{kg})$ & $25.98 \pm 0.855$ & $23.68 \pm 0.69$ & 0.091 \\
\hline Body mass index $\left(\mathrm{kg} / \mathrm{m}^{2}\right)$ & $19.4 \pm 2.608$ & $18.13 \pm 1.908$ & 0.894 \\
\hline Percent body fat $(\%)$ & $0.86 \pm 0.0255$ & $0.828 \pm 0.013$ & 0.351 \\
\hline Waist-hip ratio & $64.82 \pm 11.6$ & $51.73 \pm 7.8$ & 0.792
\end{tabular}

Values are expressed as means \pm standard errors of the means. 
Table 2. Comparison of the blood lactate levels between the two groups \& difference values by sets within a group

\begin{tabular}{|c|c|c|c|c|}
\hline HIIE Set & Time & $\begin{array}{l}\text { Control group } \\
\qquad(n=5)\end{array}$ & $\begin{array}{l}\text { Lactate group } \\
\qquad(n=6)\end{array}$ & $P$ value \\
\hline \multirow{5}{*}{$1^{\text {st }}$} & Pre-exercise & $1.56 \pm 0.258$ & $1.7 \pm 0.308$ & 0.346 \\
\hline & Post-exercise & $9.04 \pm 2.079$ & $10.73 \pm 1.536$ & 0.154 \\
\hline & Post-exercise $10 \mathrm{~min}$ & $5.96 \pm 1.481$ & $7.383 \pm 1.69$ & 0.214 \\
\hline & Post-exercise $20 \mathrm{~min}$ & $3.52 \pm 0.677$ & $5.417 \pm 1.059$ & 0.123 \\
\hline & Post-exercise $30 \mathrm{~min}$ & $2.9 \pm 0.281$ & $4.683 \pm 1.046$ & 0.102 \\
\hline \multirow{5}{*}{$2^{\text {nd }}$} & Pre-exercise & $2.18 \pm 0.287$ & $3.083 \pm 0.572$ & 0.178 \\
\hline & Post-exercise & $7.68 \pm 1.254$ & $9.083 \pm 1.908$ & 0.396 \\
\hline & Post-exercise $10 \mathrm{~min}$ & $4.1 \pm 0.764$ & $6.167 \pm 1.098$ & 0.082 \\
\hline & Post-exercise $20 \mathrm{~min}$ & $3 \pm 0.370$ & $5.867 \pm 1.283$ & 0.097 \\
\hline & Post-exercise $30 \mathrm{~min}$ & $2.58 \pm 0.338$ & $3.967 \pm 0.779$ & 0.141 \\
\hline \multirow{5}{*}{$\begin{array}{l}\text { Comparison of } \\
\text { changes in the values } \\
\text { according to sets of } \\
\text { HIIE between groups } \\
\left(1^{\text {st }}-2^{\text {nd }}\right)\end{array}$} & Pre-exercise & $-0.62 \pm 0.338$ & $-1.383 \pm 0.492$ & 0.119 \\
\hline & Post-exercise & $1.36 \pm 1.03$ & $1.65 \pm 0.828$ & 0.251 \\
\hline & Post-exercise $10 \mathrm{~min}$ & $1.86 \pm 1.054$ & $1.217 \pm 0.702$ & 0.444 \\
\hline & Post-exercise 20 min & $0.52 \pm 0.35$ & $-0.45 \pm 0.515$ & 0.082 \\
\hline & Post-exercise $30 \mathrm{~min}$ & $0.32 \pm 0.24$ & $0.717 \pm 0.626$ & 0.124 \\
\hline
\end{tabular}

Mann-Whitney test; values are expressed as means \pm standard errors of the means.

comparing the value of changes by set within the groups at each time point, the blood lactate level of the lactate group was lower than that of the control group $(P=0.0823) 20$ min after HIIE. Blood lactate levels in both the groups were higher in the first HIIE set than those in the second set in most of the time points post exercise. However, the blood lactate level of the lactate group was only higher in the second HIIE set than that in the first HIIE set at 20 min after HIIE.

\section{Executive function}

To check the effect of lactate consumption on EF after acute repeated HIIE, the interference scores were examined using CWST. The overall changes in the blood lactate levels of the groups are presented in Figure 2. The changes in the interference scores over time in each group are shown in Table S5. There were no significant differences according to time in the interference scores of the first and second sets in either group $(P>0.05)$. Although the interference scores tended to change over time in the first set of the lactate group $(P=0.0504)$, the post-hoc test showed that there was no significant difference during all the post-exercise periods compared to the pre-exercise period $(P>0.05)$.

Furthermore, the comparison of interference scores between the two groups and that of changes in the interference scores according to the sets in each group are shown by time points in Table 3. There were no significant differences in the interference scores of the two groups $(P>0.05)$. However, we observed that the interference score of the lactate group in the two sets was significantly lower than that of the control group 20 min after HIIE $(P<0.05)$.

The results of the accuracy rate and total reaction time of the CWST are presented in Supplementary Tables S6-S9. In particular, we observed that the change in the accuracy rate of the lactate group within the two sets was significantly lower than that in the control group 20 min after HIIE $(P<$ 0.05 ), as presented in Supplementary Table S7. Moreover, the reaction time showed a significant change over time in

Table 3. Comparison of the interference scores between the two groups \& difference values by sets within a group

\begin{tabular}{|c|c|c|c|c|}
\hline HIIE Set & Time & $\begin{array}{l}\text { Control group } \\
\qquad(n=5)\end{array}$ & $\begin{array}{l}\text { Lactate group } \\
\qquad(n=6)\end{array}$ & $P$ value \\
\hline \multirow{5}{*}{$1^{\text {st }}$} & Pre-exercise & $22.19 \pm 8.326$ & $20.85 \pm 6.205$ & 0.5 \\
\hline & Post-exercise & $21.08 \pm 9.066$ & $9.456 \pm 3.408$ & 0.2143 \\
\hline & Post-exercise $10 \mathrm{~min}$ & $17.94 \pm 8.041$ & $18.18 \pm 5.225$ & 0.465 \\
\hline & Post-exercise $20 \mathrm{~min}$ & $14.84 \pm 8.521$ & $18.46 \pm 3.284$ & 0.465 \\
\hline & Post-exercise $30 \mathrm{~min}$ & $19.52 \pm 7.401$ & $19.36 \pm 3.992$ & 0.465 \\
\hline \multirow{5}{*}{$2^{\text {nd }}$} & Pre-exercise & $24.49 \pm 6.403$ & $14.68 \pm 6.655$ & 0.268 \\
\hline & Post-exercise & $23.15 \pm 11.33$ & $10.73 \pm 3.474$ & 0.214 \\
\hline & Post-exercise $10 \mathrm{~min}$ & $17.53 \pm 7.111$ & $15.87 \pm 5.367$ & 0.465 \\
\hline & Post-exercise $20 \mathrm{~min}$ & $37.05 \pm 12.82$ & $23.12 \pm 2.587$ & 0.331 \\
\hline & Post-exercise $30 \mathrm{~min}$ & $19.49 \pm 4.616$ & $16.18 \pm 6.619$ & 0.268 \\
\hline \multirow{5}{*}{$\begin{array}{l}\text { Comparison of } \\
\text { changes in the values } \\
\text { according to sets of } \\
\text { HIIE between groups } \\
\left(1^{\text {st }}-2^{\text {nd }}\right)\end{array}$} & Pre-exercise & $-2.302 \pm 9.879$ & $6.174 \pm 6.36$ & 0.268 \\
\hline & Post-exercise & $-2.064 \pm 14.97$ & $-1.27 \pm 3.34$ & 0.465 \\
\hline & Post-exercise $10 \mathrm{~min}$ & $0.4104 \pm 12.36$ & $2.31 \pm 4.667$ & 0.465 \\
\hline & Post-exercise $20 \mathrm{~min}$ & $-22.2 \pm 5.99$ & $-4.657 \pm 2.213$ & $P<0.05$ \\
\hline & Post-exercise $30 \mathrm{~min}$ & $-0.237 \pm 8.323$ & $3.181 \pm 4.592$ & 0.5 \\
\hline
\end{tabular}

Mann-Whitney test; values are means \pm standard errors of the means. 


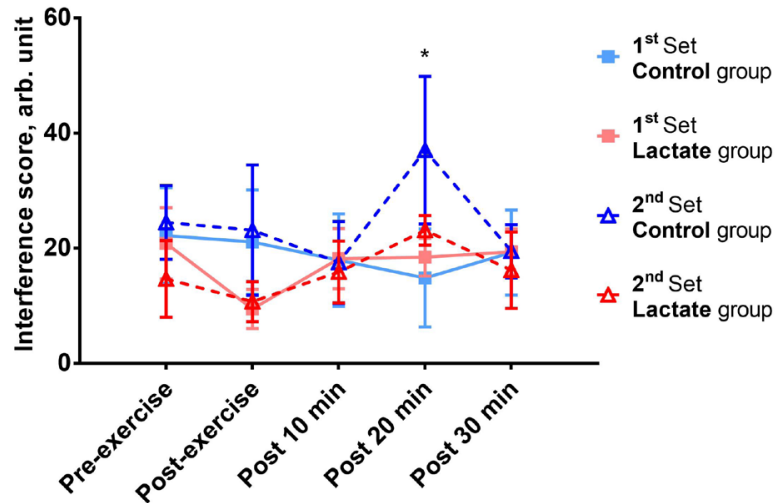

Figure 2. Changes in Stroop test scores; the inter-group difference in the interference scores in the $2^{\text {nd }}$ HIIE set and the variation over the set within each group $\left(1^{\text {st }}\right.$ set $-2^{\text {nd }}$ set value $)$ were compared over time using a Mann-Whitney test. Friedman test was conducted to check the time-induced change within each group by set. Data are expressed as the means \pm standard errors of the means. ${ }^{*} p<0.05$ indicates a significant difference between the two groups in changes in the interference score of each group within the two sets.

the second set of the lactate group $(P<0.05)$, although there was no significant difference between the two groups $(P>$ 0.05) (Supplementary Tables S8-S9).

\section{Other measurement variables}

The results of blood and cardiovascular variables are presented in Supplementary Tables S10-S15. The BDNF level in the lactate group was higher than that in the control group at pre-exercise in the first set of HIIE $(P<0.05)$. However, there were no significant changes over time and between the two groups in the second set $(P>0.05)$, as presented in Supplementary Tables S16-S17.
The results of psychological variables are presented in Supplementary Tables S18-S23. At $10 \mathrm{~min}$ and $20 \mathrm{~min}$ after the $2^{\text {nd }}$ HIIE $(P<0.05)$, the lactate group had lower arousal levels than the control group (Supplementary Table S19). However, on comparing the value of changes by sets within the groups in the post-exercise period, there were no significant differences in the arousal levels between the two groups. In addition, there were no significant differences in mental fatigue between the two groups $(P>0.05)$ (Supplementary Table S21).

\section{Correlation between blood lactate and the results of the CWST}

The correlation analysis between the average blood lactate level and the interference score of each group at specific time points is illustrated in Figure 3. The blood lactate levels showed a significant positive correlation with the results of the CWST. The blood lactate concentration was negatively correlated with the interference score $(r=-0.394$ and $P<0.05)$ (Figure 3A) and total reaction time $(r=-0.6466$ and $P<0.01$ ) (Figure 3B).

\section{DISCUSSION}

A single bout of HIIE can temporarily increase blood lactate levels, brain lactate uptake, and EF in adult males. However, this effect of exercise-induced increment was attenuated after repeated HIIE. Moreover, several studies have suggested a correlation between lactate level and EF, pointing to possible causation effects of lactate on $\mathrm{EF}^{7,20}$. Based on this previous research, we aimed to identify the effect of lactate consumption in maintaining the reduced EF after repeated HIIE. Our results confirm the positive correlation between lactate intake on the maintenance of the reduced $\mathrm{EF}$ and the causal relationship between lactate consumption
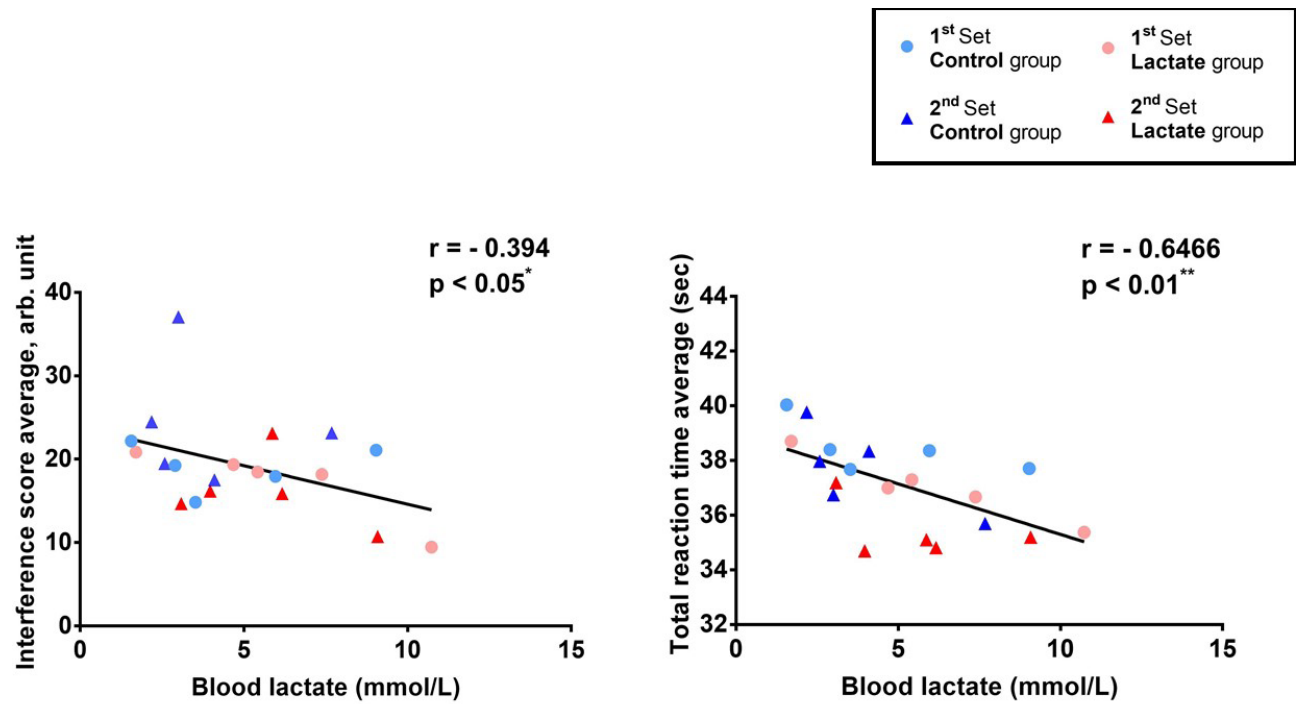

Figure 3. The correlation between blood lactate levels and the results of the CWST; (A) interference score and (B) total reaction time of CWST. The average of the blood lactate level and the CWST results for each group of each time point were calculated to analyze the correlation between the two variables. 
and $\mathrm{EF}$.

In this study, the EF of participants was analyzed using interference scores, response times, and accuracies measured by the CWST. The interference score (an indicator of EF) represents the ability to inhibit automated cognitive processes in response to specific stimuli and that to perform lessautomated cognitive processes. Twenty minutes after HIIE, changes in the interference score and accuracy between sets in the lactate-consuming group were significantly lower and higher than those in the control group, respectively. Additionally, unlike the control group, the reaction time of the lactate group improved $30 \mathrm{~min}$ after exercise. Therefore, the overall CWST results in the lactate group improved compared to that in the control group. These results are in line with those of previous studies, such as that by Holloway et al. $^{28}$, who reported improvements in cognitive recovery with $100 \mathrm{mM}$ L-lactate injections in rats in traumatic brain injury models. Moreover, Bisri et al. ${ }^{30}$ suggested that exogenous hyperosmolar sodium lactate infusions before surgery can improve neurocognitive function in patients with mild traumatic brain injury. Therefore, we also suggest that lactate consumption has a favorable effect on the reduction of EF due to repeated HIIE.

The blood lactate levels in both the groups reduced more significantly after the second HIIE set than after the first set. This result is consistent with previous studies ${ }^{7,20}$ showing reduced blood lactate levels due to repeated HIIE. Additionally, this phenomenon has also been observed among the blood lactate levels of players after the first and second half of football matches, in which the players have similar physical demands after repeated HIIE ${ }^{21}$. High-intensity or long-term exercise gradually depletes the energy sources in the body such as ATP, creatine phosphate, and glycogen. The depletion of glucose and glycogen, precursors of lactate, suggests decreases in lactate production and blood lactate level. It also suggests an energy gap greater than that normally needed to transmit ions and neurotransmitters for neural activity, which can be a causative factor of central fatigue ${ }^{31}$.

While previous studies have reported increases in blood lactate levels after injection ${ }^{13,16}$, a few studies have examined changes in lactate levels resulting from lactate consumption. In this study, the decrease in the blood lactate levels due to repeated HIIE was balanced through lactate consumption. These results suggest that the level of brain lactate uptake can be maintained, as results from previous studies also showed that brain lactate uptake increases in proportion to blood lactate levels ${ }^{3,16,32}$.

Additionally, the higher the exercise intensity, the greater the proportion of lactate in brain metabolism ${ }^{17}$, which suggests an increase in the brain's lactic acid consumption during exercise. Taken together, repeated HIIE not only increased the rate of lactate metabolism in the brain but it also gradually depleted energy. This requirement would have been maintained by the supplementation of the insufficient blood lactate levels and enabling brain lactate uptake through oral consumption. In other words, it may mediate the fatigue and reduction of EF induced by the reduction of lactate required for the transport of neurotransmitters and ions during neural activity $7,33-35$. This interpretation is supported by our results, which show that the timings of the increase in blood lactate levels and that of differences in EF are consistent at $20 \mathrm{~min}$ after exercise. Additionally, as reported in previous studies ${ }^{7,20}$, the level of blood lactate is the only variable that correlated with EF.

BDNF is a member of the neurotrophin family of growth factors that enables neurogenesis, neuroprotection, and neuroplasticity ${ }^{36,37}$. Therefore, it is one of the most commonly used indicators for investigating improvements in the cognitive function due to exercise. In a previous study, not only was the correlation between BDNF, a major biomarker of cognitive function, and lactate presented but also the possibility of lactate mediating BDNF signaling was suggested. The increased levels of lactate and BDNF in the blood were correlated $^{38}$, lactic acid injection at rest increased the BDNF level in the blood ${ }^{39}$, and lactate treatment in a rodent model of brain damage induced increases in BDNF and improved the cognitive performance ${ }^{13}$. However, unlike the results of previous studies, our results did not show significant changes in blood BDNF due to lactate intake and exercise. Based on recent results, BDNF does not always increase after exercise. Maderova et al. ${ }^{40}$ suggested that acute exercise increased serum BDNF but not plasma BDNF. Therefore, we should have examined BDNF levels in serum as well as plasma. In addition, blood BDNF levels did not significantly correlate with the Stroop test results. Therefore, as presented by Hashimoto et al. ${ }^{7}$, we considered that BDNF did not have a significant impact on the improvement of EF.

In a previous study, the increase in the level of arousal after exercise showed a positive correlation with the increase in EF measured by the Stroop test ${ }^{27}$. In this study, the increase in arousal level after HIIE compared with that before HIIE was consistent with previous studies. However, our results showed that the arousal level of the control group was significantly higher than that of the lactate group whereas the EF of the lactate group was more improved than that of the control group. Furthermore, we expected that the mental fatigue will also decrease due to the improvement of central fatigue through lactate consumption. However, there was no significant difference between the lactate and control groups, and there was no significant correlation between EF and mental fatigue. However, as demonstrated by Pageaux et al. ${ }^{41}$, it could be considered that changes in mental fatigue levels do not necessarily indicate changes in central fatigue. It is known that patients with attention deficit hyperactivity disorder, which involves a general lack of concentration, have impaired $\mathrm{EF}^{42}$. However, our results showed that the $\mathrm{EF}$ of the lactate group was higher than that of the control group, although the concentration level of the lactate group was lower than that of the control group. In addition, there was no significant correlation between the concentration level and the EF. In other words, there was no significant correlation between psychological changes and EF, although several results of the psychological variables in this study showed different tendencies from those of previous studies. Therefore, we considered that psychological variables do 
not significantly impact the improvement of EF.

However, this study has limitations, and further studies are required to address those without bias. First, the increase in the blood lactate level in the experimental group was not statistically significant, and the metabolic pathway of lactate was not identified. Therefore, we are unable conclude if the ingested lactate had been transferred to the brain and was used to improve EF. Additionally, the small sample size of this study limits its statistical power. The complexity of the experiment process, which takes about two and a half hours to measure 10 variables, and the high intensity of exercise caused many people to drop out. Sufficient participants are needed to achieve adequate statistical power, and a crossover design would perhaps be more suitable than our current design. Lactate consumption methods in this study were also limited because of the lack of advanced empirical studies on the proper concentration and timing of lactate beverage consumption. For this reason, the experimental beverage manufacturing process was established through pilot test results, as presented in Supplementary Tables S1 and S2. Further studies should be conducted based on more appropriate concentrations and timings of lactate intake.

Nonetheless, the results of this study are meaningful, given the lack of research of cognitive improvement on lactate consumption. Additionally, our study revealed a causal relationship between lactate consumption and EF that was significantly different from the correlation found in previous studies. Therefore, the results of this study could provide meaningful data for research on the improvement of cognitive performance of athletes and clinical research related to cognitive diseases.

\section{ACKNOWLEDGEMENTS}

All procedures were approved by the ethics committee of Seoul National University (IRB No. 1909/002-010). The participants provided written informed consent to participate in this study. This research was supported by the National Research Foundation (700-20190019 and NRF2020R1C1C1006414).

\section{REFERENCES}

1. Katakam A. Data check: a historical breakdown of when teams score at the Fifa World Cup. 2018 2018.07.05 2019.04.14]; Available from: https://scroll.in/field/884136/data-check-a-historicalbreakdown-of-when-teams-score-at-the-fifa-world-cup.

2. DE Oliveira MC, Orbetelli R, DE Barros Neto TL. Call accuracy and distance from the play: a study with Brazilian soccer referees. Int J Exerc Sci. 2011;4:30-8.

3. Bouzat P, Sala N, Suys T, Zerlauth JB, Marques-Vidal P, Feihl F, Bloch J, Messerer M, Levivier M, Meuli R, Magistretti PJ, Oddo M. Cerebral metabolic effects of exogenous lactate supplementation on the injured human brain. Intensive Care Med. 2014;40:41221.

4. Huijgen BC, Leemhuis S, Kok NM, Verburgh L, Oosterlaan J,
Elferink-Gemser MT, Visscher C. Cognitive functions in elite and sub-elite youth soccer players aged 13 to 17 years. PLoS One. 2015;10:e0144580.

5. Sakamoto S, Takeuchi H, Ihara N, Ligao B, Suzukawa K. Possible requirement of executive functions for high performance in soccer. PLoS One. 2018;13:e0201871.

6. Vestberg T, Gustafson R, Maurex L, Ingvar M, Petrovic P. Executive functions predict the success of top-soccer players. PLOS One. 2012;7:e34731.

7. Hashimoto T, Tsukamoto H, Takenaka S, Olesen ND, Petersen LG, Sorensen H, Nielsen HB, Secher NH, Ogoh S. Maintained exercise-enhanced brain executive function related to cerebral lactate metabolism in men. FASEB J. 2018;32:1417-27.

8. Scarpina F, Tagini S. The stroop color and word test. Front Psychol. 2017;8:557.

9. Abd-Elfattah HM, Abdelazeim FH, Elshennawy S. Physical and cognitive consequences of fatigue: a review. $J$ Adv Res. 2015;6:351-8.

10. Zajac A, Chalimoniuk M, Maszczyk A, Golas A, Lngfort J. Central and peripheral fatigue during resistance exercise - a critical review. J Hum Kinet. 2015;49:159-69.

11. Kluger BM, Krupp LB, Enoka RM. Fatigue and fatigability in neurologic illnesses: proposal for a unified taxonomy. Neurology. 2013;80:409-16.

12. Pattyn N, Van Cutsem J, Dessy E, Mairesse O. Bridging exercise science, cognitive psychology, and medical practice: is "cognitive fatigue" a remake of "the emperor's new clothes"? Front Psychol. 2018:9:1246.

13. Brooks GA. The science and translation of lactate shuttle theory. Cell Metab. 2018;27:757-85.

14. Bélanger M, Allaman I, Magistretti PJ. Brain energy metabolism: focus on astrocyte-neuron metabolic cooperation. Cell Metab. 2011;14:724-38.

15. Bouzier-Sore AK, Voisin P, Bouchaud V, Bezancon E, Franconi $\mathrm{JM}$, Pellerin L. Competition between glucose and lactate as oxidative energy substrates in both neurons and astrocytes: a comparative NMR study. Eur J Neurosci. 2006;24:1687-94.

16. van Hall G, Strømstad M, Rasmussen P, Jans O, Zaar M, Gam C, Quistorff B, Secher NH, Nielsen HB. Blood lactate is an important energy source for the human brain. J Cereb Blood Flow Metab. 2009:29:1121-9.

17. Smith KJ, Ainslie PN. Regulation of cerebral blood flow and metabolism during exercise. Exp Physiol. 2017;102:1356-71.

18. Kemppainen J, Aalto S, Fujimoto T, Kalliokoski KK, Långsjö J, Oikonen V, Rinne J, Nuutila P, Knuuti J. High intensity exercise decreases global brain glucose uptake in humans. J Physiol. 2005;568:323-32

19. Tsukamoto H, Suga T, Takenaka S, Tanaka D, Takeuchi T, Hamaoka T, Isaka T, Hashimoto T. Greater impact of acute high-intensity interval exercise on post-exercise executive function compared to moderate-intensity continuous exercise. Physiol Behav. 2016;155:224-30

20. Tsukamoto H, Suga T, Takenaka S, Tanaka D, Takeuchi T, Hamaoka T, Isaka T, Ogoh S, Hashimoto T. Repeated high-intensity interval exercise shortens the positive effect on executive function during post-exercise recovery in healthy young males. Physio Behav. 2016;160:26-34.

21. Stølen T, Chamari K, Castagna C, Wisløff U. Wisloff. Physiology of 
soccer: an update. Sports Med. 2005;35:501-36.

22. Russell M, West DJ, Harper LD, Cook CJ, Kilduff LP. Halftime strategies to enhance second-half performance in teamsports players: a review and recommendations. Sports Med. 2015;45:353-64.

23. Nybo L, Secher NH. Cerebral perturbations provoked by prolonged exercise. Prog Neurobiol. 2004;72:223-61.

24. McMorris T. Developing the catecholamines hypothesis for the acute exercise-cognition interaction in humans: lessons from animal studies. Physiol Behav. 2016;165:291-9.

25. Grego F, Vallier JM, Collardeau M, Bermon S, Ferrari P, Candito $\mathrm{M}$, Bayer $\mathrm{P}$, Magnié MN, Brisswalter J. Effects of long duration exercise on cognitive function, blood glucose, and counterregulatory hormones in male cyclists. Neurosci Lett. 2004;364:76-80.

26. Shields GS, Bonner JC, Moons WG. Does cortisol influence core executive functions? A meta-analysis of acute cortisol administration effects on working memory, inhibition, and set-shifting. Psychoneuroendocrinology. 2015;58:91-103.

27. Byun K, Hyodo K, Suwabe K, Ochi G, Sakairi Y, Kato M, Dan I, Soya $\mathrm{H}$. Positive effect of acute mild exercise on executive function via arousal-related prefrontal activations: an fNIRS study. Neurolmage. 2014;98:336-45.

28. Holloway R, Zhou Z, Harvey HB, Levasseur JE, Rice AC, Sun D, Hamm RJ, Bullock MR. Effect of lactate therapy upon cognitive deficits after traumatic brain injury in the rat. Acta Neurochir (Wien). 2007;149:919-27.

29. Warburton DE, Jamnik V, Bredin SS, Shephard RJ, Gledhill N. The 2019 physical activity readiness questionnaire for everyone (PAR$\mathrm{Q}+$ ) and electronic physical activity readiness medical examination (ePARmed-X+). The Health \& Fitness Journal of Canada. 2018;11:80-3.

30. Bisri T, Utomo BA, Fuadi I. Exogenous lactate infusion improved neurocognitive function of patients with mild traumatic brain injury. Asian J Neurosurg. 2016;11:151-9.

31. Matsui T, Omuro H, Liu YF, Soya M, Shima T, McEwen BS, Soya H. Astrocytic glycogen-derived lactate fuels the brain during exhaustive exercise to maintain endurance capacity. Proc Natl Acad Sci U S A. 2017;114:6358-63.

32. Rasmussen P, Wyss MT, Lundby C. Cerebral glucose and lactate consumption during cerebral activation by physical activity in humans. FASEB J. 2011;25:2865-73.

33. Barros, LF. Metabolic signaling by lactate in the brain. Trends Neurosci. 2013;36:396-404.

34. Smith D, Pernet A, Hallett WA, Bingham E, Marsden PK, Amiel SA. Lactate: a preferred fuel for human brain metabolism in vivo. $J$ Cereb Blood Flow Metab. 2003;23:658-64.

35. Hu Y, Wilson GS. A temporary local energy pool coupled to neuronal activity: fluctuations of extracellular lactate levels in rat brain monitored with rapid-response enzyme-based sensor. J Neurochem. 1997;69:1484-90.

36. Levy MJF, Boulle F, Steinbusch HW, van den Hove DLA, Kenis G, Lanfumey L. Neurotrophic factors and neuroplasticity pathways in the pathophysiology and treatment of depression. Psychopharmacology (Berl). 2018;235:2195-220.

37. Bathina S, Das UN. Brain-derived neurotrophic factor and its clinical implications. Arch Med Sci. 2015;11:1164-78.

38. Ferris $\mathrm{LT}$, Williams JS, Shen $\mathrm{CL}$. The effect of acute exercise on serum brain-derived neurotrophic factor levels and cognitive func- tion. Med Sci Sports Exerc. 2007;39:728-34.

39. Schiffer T, Schulte S, Sperlich B, Achtzehn S, Fricke H, Strüder HK. Lactate infusion at rest increases BDNF blood concentration in humans. Neurosci Lett. 2011;488:234-7.

40. Máderová D, Krumpolec P, Slobodová L, Schön M, Tirpáková V, Kovaničová Z, Klepochová R, Vajda M, Šutovský S, Cvečka J, Valkovič L, Turčáni P, Krššák M, Sedliak M, Tsai CL, Ukropcová B, Ukropec J. Acute and regular exercise distinctly modulate serum, plasma and skeletal muscle BDNF in the elderly. Neuropeptides. 2019;78:101961.

41. Pageaux B, Marcora SM, Rozand V, Lepers R. Mental fatigue induced by prolonged self-regulation does not exacerbate central fatigue during subsequent whole-body endurance exercise. Front Hum Neurosci. 2015;9:67.

42. Shuai L, Daley D, Wang YF, Zhang JS, Kong YT, Tan X, Ji N. Ji. Executive function training for children with attention deficit hyperactivity disorder. Chin Med J. (Engl). 2017;130:549-58. 